\title{
Shimura Curves for Level-3 Subgroups of the $(2,3,7)$ Triangle Group and Some Other Examples
}

\section{Citation}

Elkies, Noam D. 2006. Shimura curves for level-3 subgroups of the $(2,3,7)$ triangle group and some other examples. In Algorithmic number theory: 7th International Symposium, ANTS-VII, Berlin, Germany, July 23-28, 2006: Proceedings, ed. Florian Hess, Sebastian Pauli, Michael Pohst, 302-316. Lecture Notes in Computer Science 4076. Berlin: Springer Verlag.

\section{Published Version}

http://dx.doi.org/10.1007/11792086_22

\section{Permanent link}

http://nrs.harvard.edu/urn-3:HUL.InstRepos:11022283

\section{Terms of Use}

This article was downloaded from Harvard University's DASH repository, and is made available under the terms and conditions applicable to Other Posted Material, as set forth at http:// nrs.harvard.edu/urn-3:HUL.InstRepos:dash.current.terms-of-use\#LAA

\section{Share Your Story}

The Harvard community has made this article openly available.

Please share how this access benefits you. Submit a story.

\section{Accessibility}




\title{
Shimura curves for level-3 subgroups of the $(2,3,7)$ triangle group and some other examples
}

\author{
Noam D. Elkies \\ Department of Mathematics, Harvard University, Cambridge, MA 02138 USA
}

\begin{abstract}
The $(2,3,7)$ triangle group is known to be associated with a quaternion algebra $A / K$ ramified at two of the three real places of $K=\mathbf{Q}(\cos 2 \pi / 7)$ and unramified at all other places of $K$. This triangle group and its congruence subgroups thus give rise to various Shimura curves and maps between them. We study the genus- 1 curves $\mathcal{X}_{0}(3)$, $\mathcal{X}_{1}(3)$ associated with the congruence subgroups $\Gamma_{0}(3), \Gamma_{1}(3)$. Since the rational prime 3 is inert in $K$, the covering $\mathcal{X}_{0}(3) / \mathcal{X}(1)$ has degree 28 , and its Galois closure $\mathcal{X}(3) / \mathcal{X}(1)$ has geometric Galois group $\operatorname{PSL}_{2}\left(\mathbf{F}_{27}\right)$. Since $\mathcal{X}(1)$ is rational, the covering $\mathcal{X}_{0}(3) / \mathcal{X}(1)$ amounts to a rational map of degree 28 . We compute this rational map explicitly. We find that $\mathcal{X}_{0}(3)$ is an elliptic curve of conductor $147=3 \cdot 7^{2}$ over $\mathbf{Q}$, as is the Jacobian $\mathcal{J}_{1}(3)$ of $\mathcal{X}_{1}(3)$; that these curves are related by an isogeny of degree 13 ; and that the kernel of the 13 -isogeny from $\mathcal{J}_{1}(3)$ to $\mathcal{X}_{0}(3)$ consists of $K$-rational points. We also use the map $\mathcal{X}_{0}(3) \rightarrow \mathcal{X}(1)$ to locate some complex multiplication $(\mathrm{CM})$ points on $\mathcal{X}(1)$. We conclude by describing analogous behavior of a few Shimura curves associated with quaternion algebras over other cyclic cubic fields.
\end{abstract}

\section{Introduction}

\subsection{Review: Quaternion algebras over $K$, Shimura modular curves, and the $(2,3,7)$ triangle group.}

Let $K$ be the field $\mathbf{Q}(\cos 2 \pi / 7)$, which is the totally real cubic field of minimal discriminant (namely, discriminant 49 ); let $O_{K}$ be the ring $\mathbf{Z}[2 \cos 2 \pi / 7]$ of algebraic integers in $K$; and let $A$ be a quaternion algebra over $K$ ramified at two of the three real places and at no other place of $K$. This algebra exists because the set of ramified places has even cardinality, and is determined uniquely up to $K$-algebra isomorphism. (See for instance $[\mathrm{Vi}$ for this and other basic results on quaternion algebras and Shimura curves, and E2 for our computational context.) All maximal orders in $A$ are conjugate because $A$ is indefinite and $O_{K}$ has narrow class number 1 ; we fix one maximal order $\varnothing \subset A$. Let $\Gamma(1)$ be the group of elements of $\varnothing$ of reduced norm 1 . Since $A$ has exactly one unramified real place, $\Gamma(1)$ embeds into $\mathrm{SL}_{2}(\mathbf{R})$ as a discrete co-compact subgroup. Let $\mathcal{H}$ be the upper half-plane, with the usual action of $\mathrm{PSL}_{2}(\mathbf{R})=\mathrm{SL}_{2}(\mathbf{R}) /\{ \pm 1\}$. The quotient of $\mathcal{H}$ by $\Gamma(1) /\{ \pm 1\}$ then has the structure of a compact Riemann 
surface, and thus of an algebraic curve over $\mathbf{C}$. In fact this quotient has a natural structure as a curve over $K$, namely the Shimura curve associated to $\Gamma(1)$. We call this Shimura curve $\mathcal{X}(1)$, in analogy with the modular elliptic curve $\mathrm{X}(1)$ of the classical theory of elliptic and modular curves: as $\mathrm{X}(1)$ parametrizes elliptic curves, $\mathcal{X}(1)$ parametrizes certain abelian varieties which we shall call "O-varieties" 1 By work of Shimura Sh1, based on the classical work of Fricke F1|F2, the group $\Gamma(1) /\{ \pm 1\} \subset \mathrm{SL}_{2}(\mathbf{R})$ is the $(2,3,7)$ triangle group (the group generated by products of pairs of reflections in the sides of a hyperbolic triangle of angles $\pi / 2, \pi / 3, \pi / 7)$. Hence $\mathcal{X}(1)$ is a curve of genus 0 with elliptic points of orders $2,3,7$. We choose the rational coordinate $t: \mathcal{X}(1) \stackrel{\sim}{\longrightarrow} \mathbf{P}^{1}$ that takes the values $0,1, \infty$ respectively at these three points; this determines $t$ uniquely (as the classical modular invariant $j$ is determined by its values $1728,0, \infty$ at the elliptic points of orders 2,3 and the cusp).

Suppose now that $\Gamma \subset \Gamma(1) /\{ \pm 1\}$ is a subgroup of finite index $d$. Then $\mathcal{X}=\mathcal{H} / \Gamma$ is a compact Riemann surface with a degree- $d$ map to $\mathcal{X}(1)$ that is unramified away from the elliptic points of $\mathcal{X}(1)$. Composing the map $\pi: \mathcal{X} \rightarrow$ $\mathcal{X}(1)$ with our isomorphism $t: \mathcal{X}(1) \stackrel{\sim}{\longrightarrow} \mathbf{P}^{1}$ yields a rational function $t \circ \pi$ of degree $d$ on $\mathcal{X}$ that is unramified except above $t=0, t=1$, and $t=\infty$. Such a rational function is often called a "Belyi function" on $\mathcal{X}$, in tribute to Belyi's theorem Bel] that a Riemann surface admits such a function if and only if it can be realized as an algebraic curve over $\overline{\mathbf{Q}}$. It will be convenient, and should cause no confusion, to use $t$ also as the notation for this rational function on $\mathcal{X}$.

In particular, if $\Gamma$ is a congruence subgroup of $\Gamma(1) /\{ \pm 1\}$ - that is, if there exists a nonzero ideal $N$ of $O_{K}$ such that $\Gamma$ contains the image in $\Gamma(1) /\{ \pm 1\}$ of the normal subgroup

$$
\Gamma(N):=\{g \in \Gamma(1) \mid g \equiv 1 \bmod N \varnothing\}
$$

- then $\mathcal{X}$ is also a Shimura modular curve, parametrizing $\mathcal{O}$-varieties with some level- $N$ structure. For example, $\Gamma(N)$ itself is a congruence subgroup, corresponding to a Shimura curve parametrizing $\mathcal{O}$-varieties with full level- $N$

\footnotetext{
1 Warning: unlike the case of Shimura curves associated with quaternion algebras over $\mathbf{Q}$, here an $\mathcal{O}$-variety is not simply a principally polarized abelian variety with endomorphisms by $\varnothing$. Indeed there can be no abelian variety $V$ with $\operatorname{End}(V) \otimes \mathbf{Q}=A$, because the set of ramified primes of $A$ neither contains nor is disjoint from the set of real places of $K$. (See for instance $\mathrm{Mu}$, specifically Thm.1 on p.192 (positivity of the Rosati involution) together with the classification in Thm.2 on p.201.) The moduli description is quite complicated (see [Sh1Ca]), and requires an auxiliary quadratic extension $K^{\prime} / K$, with the field $K^{\prime}$ totally imaginary; for instance, for our case $K=\mathbf{Q}(\cos 2 \pi / 7)$ we may take $K^{\prime}=K(\sqrt{-7})=\mathbf{Q}\left(e^{2 \pi i / 7}\right)$. The moduli description yields $\mathcal{X}(1)$ as a curve over $K^{\prime}$, but fortunately $\mathcal{X}(1)$ descends to a curve over $K$ independent of the choice of $K^{\prime}$. The same is true of the curves $\mathcal{X}(N), \mathcal{X}_{1}(N), \mathcal{X}_{0}(N)$ and the maps $\mathcal{X}(N) \rightarrow \mathcal{X}_{1}(N) \rightarrow \mathcal{X}_{0}(N) \rightarrow \mathcal{X}(1)$ (to be introduced soon) that concern us in this paper, at least provided that the extension $K^{\prime} / K$ satisfies some local conditions at the primes dividing $N$. I am grateful to Benedict Gross for explaining these subtleties and suggesting the references to $\mathrm{Ca} \mid \mathrm{Mu}$. Fortunately very little of this difficult theory is needed for our computations.
} 
structure. We call this curve $\mathcal{X}(N)$, again in analogy with the classical case. If $N$ is a prime ideal with residue field $k$, the ring $\varnothing / N \varnothing$ is isomorphic with the ring $M_{2}(k)$ of $2 \times 2$ matrices over $k$, because $A$ is unramified at all finite places of $k$, including $N$. We choose one isomorphism $\iota: \varnothing / N \varnothing \stackrel{\sim}{\longrightarrow} M_{2}(k)$; the choice does not matter because all are equivalent under conjugation by $\emptyset^{*}$. We then obtain congruence subgroups $\Gamma_{0}(N) \triangleright \Gamma_{1}(N) \supset \Gamma(N)$, where $\Gamma_{0}(N)$ consists of those $g \in \Gamma(1)$ for which $\iota(g)$ is upper triangular, and $\Gamma_{1}(N)$ is the kernel of the map from $\Gamma_{0}(N)$ to $k^{*} /\{ \pm 1\}$ taking $g$ to the top left entry of the matrix $\iota(g) \bmod N \varnothing$. The corresponding Shimura curves are naturally denoted $\mathcal{X}_{0}(N)$, $\mathcal{X}_{1}(N)$ respectively. Once one has formulas for the cover $\mathcal{X}_{0}(N) \rightarrow \mathcal{X}(1)$, one can use them as in Se to obtain explicit extensions of $K$ or $\mathbf{Q}$ (the latter when the residual characteristic of $N$ does not split in $O_{K}$ ) whose normal closures have Galois groups $\mathrm{PSL}_{2}(k)$, Aut $\left(\mathrm{PGL}_{2}(k)\right)$, or other groups intermediate between these two.

Analogous to the Atkin-Lehner involution $w_{N}$ of the classical modular curve $\mathrm{X}_{0}(N)$, we have an involution $w_{N}$ of $\mathcal{X}_{0}(N)$, which can be constructed either from the normalizer of $\Gamma_{0}(N)$ in $A^{*}$ or by invoking the dual isogeny of the "cyclic" isogeny between $\mathcal{O}$-varieties parametrized by a generic point of $\Gamma_{0}(N)$. Once explicit formulas are known for both the map $\mathcal{X}_{0}(N) \rightarrow \mathcal{X}(1)$ and the involution $w_{N}$, one can easily compute other interesting data. By eliminating $p \in \mathcal{X}_{0}(N)$ from the system $T_{1}=t(p), T_{2}=t\left(w_{N}(p)\right)$, we obtain the "modular polynomial" $\Phi_{N}\left(T_{1}, T_{2}\right)$, which is the irreducible polynomial such that $\Phi_{N}\left(t_{1}, t_{2}\right)=0$ if and only if $t_{1}, t_{2} \in \mathbf{C}$ are $t$-coordinates of " $N$-isogenous" points on $\mathcal{X}(1)$. The solutions of $\Phi_{N}(t, t)=0$ are then coordinates of points of complex multiplication $(\mathrm{CM})$ on $\mathcal{X}(1)$, that is, points that parametrize $\mathcal{O}$-varieties with CM. Finally, with some more effort we can obtain equations defining the recursive tower of curves $\mathcal{X}_{0}\left(N^{n}\right)(n=1,2,3, \ldots)$, whose reduction modulo any prime $\varpi$ of $O_{K}$ not dividing $N$ is asymptotically optimal over the quadratic extension of the residue field of $\varpi$ (see [E1]).

If $\Gamma$ is a proper normal subgroup, congruence or not, in $\Gamma(1) /\{ \pm 1\}$, then the quotient group $G$ acts on $\mathcal{X}$. By the Riemann-Hurwitz formula, $d=|G|=$ $84(g-1)$, where $g$ is the genus of $\mathcal{X}$. That is, $(\mathcal{X}, G)$ is a "Hurwitz curve": a curve of genus $g>1$ that attains the Hurwitz bound ( $[\mathrm{H}$, see also $[\mathrm{ACGH}$, Ch.I, Ex.F-3 ff., pp.45-47]) on the number of automorphisms of a curve of genus $g$ over a field of characteristic zero. Conversely, all Hurwitz curves arise in this way for some proper normal subgroup of $\Gamma(1)$. For example, if $N$ is a prime ideal with residue field $k$ then $\mathcal{X}(N)$ is a curve with $G=\mathrm{PSL}_{2}(k)$ that attains the Hurwitz bound.

The first few possibilities for $N$ yield $k$ of size $7,8,13$, and 27 . The first of these, with $N$ the prime of $K$ above the totally ramified rational prime 7 , yields the famed Klein quartic of genus 3, which also arises as the classical modular curve $\mathrm{X}(7)$ (see [E3]). The second, with $N$ above the inert rational prime 2, yields the Fricke-Macbeath curve of genus 7 F3|Ma]. If $N$ is any of the three primes above the split rational prime 13 then $\mathcal{X}(N)$ is a curve of genus 14 that 
can be defined over $K$ but not over $\mathbf{Q}\left[\mathbf{S t r}\right.$. In each of those cases, $\mathcal{X}_{0}(N)$ has genus zero.

The next case, and the main focus of this paper, is the prime of residue field $\mathbf{F}_{27}$ above the inert rational prime 3 . We call the resulting Shimura curves $\mathcal{X}_{0}(3)$, $\mathcal{X}_{1}(3), \mathcal{X}(3)$. Since this prime is invariant under $\operatorname{Gal}(K / \mathbf{Q})$, these Shimura curves and their Belyi maps to $\mathcal{X}(1)$ (though not the action of $\mathrm{PSL}_{2}\left(\mathbf{F}_{27}\right)$ on $\mathcal{X}(3)$ ) can be defined over $\mathbf{Q}\left[\mathrm{DN} / \mathrm{WO}_{0}\right.$. Here for the first time $\mathcal{X}_{0}(N)$ has positive genus; we calculate that $\mathcal{X}_{0}(3)$ and $\mathcal{X}_{1}(3)$ both have genus 1 . The determination of the curve $\mathcal{X}_{0}(3)$, and of its degree-28 Belyi map to $\mathcal{X}(1)$ - that is, the degree-28 function $t$ on $\mathcal{X}_{0}(3)$ - requires techniques beyond those of $\mathrm{E} 2$.

In section 2 of this paper, we exhibit equations for this cover and show how we compute them. We locate the coefficients to high $p$-adic precision via Newton's method, using $p=29$, a prime at which the cover has good reduction but some of the coefficients are known in advance modulo $p$ and the rest can be determined algebraically. We then compute the simplest rational numbers consistent with the $p$-adic approximations, and prove that they are in fact the correct coefficients. The same method, possibly extended by exhaustive searching mod $p$, can be used to compute other such covers. (For instance, we used it to compute a previously unknown Shimura-Belyi function of degree 26 connected with the $(2,3,8)$ triangle group. We shall give the details of this computation, and other Shimura-Belyi maps for the $(2,3,8)$ triangle group, in a later paper.)

Having obtained equations for the map $\mathcal{X}_{0}(3) \rightarrow \mathcal{X}(1)$, we use those equations as explained above to locate several CM points on $\mathcal{X}(1)$. In particular, we confirm our conjecture from E2] for the rational point of CM discriminant -11: we had computed its $t$-coordinate to high enough precision to convincingly guess it as an element of $\mathbf{Q}$, but could not prove this guess. It also follows immediately from our formulas that $\mathcal{X}_{0}(3)$ has a $\mathbf{Q}$-rational point, and is thus an elliptic curve. In section 3 of this paper, we note some arithmetic properties of this curve, and use the modular structure to explain them. In particular, $\mathcal{X}_{0}(3)$ admits a rational 13 -isogeny to another elliptic curve, which we identify as the Jacobian of $\mathcal{X}_{1}(3)$. We then also explain why the kernel of the dual isogeny from this Jacobian to $\mathcal{X}_{0}(3)$ must consist of $K$-rational points, a fact first noted via direct computation by Mark Watkins [Wa].

In the final section we give some other examples suggested by these ideas. Watkins later found in the tables of elliptic curves two pairs of 7 -isogenous curves, of conductors $162=2 \cdot 9^{2}$ and $338=2 \cdot 13^{2}$, with 7 -torsion structure over the cyclic cubic fields of discriminant $9^{2}$ and $13^{2}$. He suggested that each of these pairs might thus be the Jacobians of the Shimura modular curves $\mathcal{X}_{0}(2), \mathcal{X}_{1}(2)$ for a quaternion algebra over the cyclic cubic field of discriminant $9^{2}, 13^{2}$ respectively that is ramified at two of its three real places and at no finite primes. We verify that this suggestion is correct for the curves of conductor $2 \cdot 9^{2}$, corresponding to a subgroup of the $(2,3,9)$ triangle group. We cannot at present compute the analogous curves and maps for the Shimura curves of level 2 associated with a quaternion algebra over the cubic field of conductor $13^{2}$, though we verify that both curves have genus 1 . It might be possible to identify the curves using more 
refined arithmetical information as in [GR problems of explicitly computing the maps $\mathcal{X}_{0}(2) \rightarrow \mathcal{X}(1)$ and $w_{2}$.

\section{The curve $\mathcal{X}_{0}(3)$}

\subsection{Preliminaries}

We use the notations of the previous section. Let $t$ be the degree-28 Belyi function on $\mathcal{X}_{0}(3)$. The elements of orders 2, 3, 7 in $\operatorname{PSL}_{2}\left(\mathbf{F}_{27}\right)$ act on $\mathbf{P}^{1}\left(\mathbf{F}_{27}\right)$ by permutations with cycle structure $2^{14}, 3^{9} 1,7^{4}$. We denote by $P_{0}$ the unique simple zero of $(t-1)$ on $\mathcal{X}_{0}(3)$ (corresponding to the unique fixed point of an element of order 3 in $\mathrm{PSL}_{2}\left(\mathbf{F}_{27}\right)$ ). Since $\mathcal{X}_{0}(3)$ and $t$ are defined over $\mathbf{Q}$, so is $P_{0}$. The preimages other than $P_{0}$ of $0,1, \infty$ under $t$ constitute disjoint effective divisors $D_{14}, D_{9}, D_{4}$, which are the sum of $14,9,4$ distinct points on $\mathcal{X}_{0}(3)$ respectively, such that

$$
t^{*}(0)=2 D_{14}, \quad t^{*}(1)=3 D_{9}+\left(P_{0}\right), \quad t^{*}(\infty)=7 D_{4} .
$$

By the Riemann-Hurwitz formula, $\mathcal{X}_{0}(3)$ is a curve of genus 1 . We give $\mathcal{X}_{0}(3)$ the structure of an elliptic curve by choosing $P_{0}$ as the origin of the group law. Since $P_{0}$ is the unique point of $\mathcal{X}_{0}(3)$ parametrizing a cyclic 3 -isogeny between the order-3 elliptic point $t=1$ of $\mathcal{X}(1)$ and itself, $P_{0}$ must be fixed under $w_{3}$. Therefore $w_{3}$ is the unique involution of $\mathcal{X}_{0}(3)$ as an elliptic curve, namely, multiplication by -1 .

Proposition 1. i) The differential dt has divisor $D_{14}+2 D_{9}-8 D_{4}$. The divisors $D_{14}, D_{9}, D_{4}$ are linearly equivalent to $14\left(P_{0}\right), 9\left(P_{0}\right), 4\left(P_{0}\right)$, respectively.

ii) There are nonzero rational functions $F_{14}, F_{9}, F_{4}$ on $\mathcal{X}_{0}(3)$ with divisors $D_{14}-14\left(P_{0}\right), D_{9}-9\left(P_{0}\right), D_{4}-4\left(P_{0}\right)$. For each choice of $F_{14}, F_{9}, F_{4}$, there exist nonzero scalars $\alpha, \beta$ such that $F_{14}^{2}=\alpha F_{4}^{7}+\beta F_{9}^{3}$ and $t=F_{14}^{2} / \alpha F_{4}^{7}$. If the $F_{n}$ are defined over $\mathbf{Q}$ then $\alpha, \beta$ are rational as well.

Proof: i) Since $\mathcal{X}_{0}(3)$ has genus one, the divisor of $d t$ is linearly equivalent to zero. This divisor is regular except for poles of order 8 at the four points of $D_{4}$. Moreover, $d t$ has simple zeros at the points of $D_{14}$ and double zeros at the points of $D_{9}$. This accounts for $14+2 \cdot 9=32$ zeros, same as the number $8 \cdot 4$ of poles, so $d t$ has no further zeros (which we could also have deduced from the fact that the map $t$ is unramified when $t \notin\{0,1, \infty\}$ ).

It follows that $D_{14}+2 D_{9} \sim 8 D_{4}$. This together with the linear equivalence of the divisors $t^{*}(0), t^{*}(1), t^{*}(\infty)$ yields our claim that $D_{n} \sim n\left(P_{0}\right)$ for each $n=14,9,4$; for instance

$$
\begin{aligned}
D_{4}= & 49 D_{4}-48 D_{4} \sim 7\left(3 D_{9}+\left(P_{0}\right)\right)-6\left(D_{14}+2 D_{9}\right) \\
& =3\left(3 D_{9}-2 D_{14}\right)+7\left(P_{0}\right) \sim-3\left(P_{0}\right)+7\left(P_{0}\right)=4\left(P_{0}\right) .
\end{aligned}
$$

ii) Functions $F_{n}$ with divisors $D_{n}-n\left(P_{0}\right)$ exist by part (i). The rational functions $t$ and $F_{14}^{2} / F_{4}^{7}$ have the same divisor, so $F_{14}^{2} / F_{4}^{7}=\alpha t$ for some nonzero scalar $\alpha$. Likewise $t-1$ and $F_{9}^{3} / F_{4}^{7}$ have the same divisor, so $F_{9}^{3} / F_{4}^{7}=\alpha_{0}(t-1)$ for some 
nonzero scalar $\alpha_{0}$. Eliminating $t$ yields the desired identity $F_{14}^{2}=\alpha F_{4}^{7}+\beta F_{9}^{3}$ with $\beta=\alpha / \alpha_{0}$. It follows that $\alpha$, and thus also $\beta$, is rational if each $F_{n}$ is defined over Q. This completes the proof of Proposition 1. $\diamond$

Now fix a nonzero holomorphic differential $\omega$ on $\mathcal{X}_{0}(3)$ defined over $\mathbf{Q}$, and define a derivation $f \mapsto f^{\prime}$ on the field of rational functions on $\mathcal{X}_{0}(3)$ by $d f=f^{\prime} \omega$.

Proposition 2. i) Let $F_{14}, F_{9}, F_{4}$ be as in Proposition 1(ii). Then $F_{14}, F_{9}^{2}$, $F_{4}^{6}$ are scalar multiples of

$$
3 F_{4} F_{9}^{\prime}-7 F_{4}^{\prime} F_{9}, \quad 2 F_{4} F_{14}^{\prime}-7 F_{4}^{\prime} F_{14}, \quad 2 F_{9} F_{14}^{\prime}-3 F_{9}^{\prime} F_{14}
$$

respectively.

ii) The functions

$$
\frac{14 F_{9} F_{4}^{\prime \prime}-13 F_{9}^{\prime} F_{4}^{\prime}}{F_{4}}, \quad \frac{6 F_{4} F_{9}^{\prime \prime}-29 F_{4}^{\prime} F_{9}^{\prime}}{F_{9}}, \quad \frac{6 F_{4} F_{14}^{\prime \prime}-43 F_{4}^{\prime} F_{14}^{\prime}}{F_{14}}
$$

on $\mathcal{X}_{0}(3)$ are regular except at $P_{0}$.

Proof: i) By Proposition 1 we have $t=F_{14}^{2} / \alpha F_{4}^{7}$. Taking the logarithmic derivative, we find

$$
\frac{d t}{t}=\left(2 \frac{F_{14}^{\prime}}{F_{14}}-7 \frac{F_{4}^{\prime}}{F_{4}}\right) \omega=\frac{2 F_{4} F_{14}^{\prime}-7 F_{4}^{\prime} F_{14}}{F_{4} F_{14}} \omega .
$$

Since $d t$ has divisor $D_{14}+2 D_{9}-8 D_{4}$, the divisor of $d t / t$ is $2 D_{9}-D_{14}-D_{4}$. Thus $F_{4} F_{14} d t / t$ is a differential with divisor $2 D_{9}-18\left(P_{0}\right)$, same as the divisor of $F_{9}^{2}$. Since the divisor of a differential on a genus-one curve is linearly equivalent to zero, it follows that $2 F_{4} F_{14}^{\prime}-7 F_{4}^{\prime} F_{14}$ is a multiple of $F_{9}^{2}$, as claimed. The formulas for $F_{14}$ and $F_{4}^{6}$ are obtained in the same way by computing the logarithmic derivatives of $t-1$ and $t /(t-1)$ respectively.

ii) Each of these is obtained by substituting one of the identities in (i) into another. Since the computations are similar and we shall use only the result concerning $\left(6 F_{4} F_{9}^{\prime \prime}-29 F_{4}^{\prime} F_{9}^{\prime}\right) / F_{9}$, we prove this result, and again leave the other two as exercises.

We have $F_{14}=\gamma_{1}\left(3 F_{4} F_{9}^{\prime}-7 F_{4}^{\prime} F_{9}\right)$ and $F_{9}^{2}=\gamma_{2}\left(2 F_{4} F_{14}^{\prime}-7 F_{4}^{\prime} F_{14}\right)$ for some nonzero scalars $\gamma_{1}, \gamma_{2}$. Hence

$$
\begin{aligned}
F_{9}^{2} & =\gamma_{1} \gamma_{2}\left(2 F_{4}\left(3 F_{4} F_{9}^{\prime}-7 F_{4}^{\prime} F_{9}\right)^{\prime}-7 F_{4}^{\prime}\left(3 F_{4} F_{9}^{\prime}-7 F_{4}^{\prime} F_{9}\right)\right) \\
& =\gamma_{1} \gamma_{2}\left(\left(6 F_{4} F_{9}^{\prime \prime}-29 F_{4}^{\prime} F_{9}^{\prime}\right) F_{4}+\left(49{F_{4}^{\prime}}^{2}-14 F_{4} F_{4}^{\prime \prime}\right) F_{9}\right) .
\end{aligned}
$$

Dividing by $F_{9}$, we find that

$$
\left(6 F_{4} F_{9}^{\prime \prime}-29 F_{4}^{\prime} F_{9}^{\prime}\right) \frac{F_{4}}{F_{9}}+\left(49{F_{4}^{\prime}}^{2}-14 F_{4} F_{4}^{\prime \prime}\right)=\frac{1}{\gamma_{1} \gamma_{2}} F_{9},
$$

which is regular away from $P_{0}$. The same is true for $49{F_{4}^{\prime}}^{2}-14 F_{4} F_{4}^{\prime \prime}$, and thus also for $\left(6 F_{4} F_{9}^{\prime \prime}-29 F_{4}^{\prime} F_{9}^{\prime}\right) F_{4} / F_{9}$. Since $F_{4}$ and $F_{9}$ have no common zeros, it follows that $\left(6 F_{4} F_{9}^{\prime \prime}-29 F_{4}^{\prime} F_{9}^{\prime}\right) / F_{9}$ has no poles except for a multiple pole at $P_{0}$, as claimed. $\diamond$ 


\subsection{Computation}

Theorem 1. The curve $\mathcal{X}_{0}(3)$ is isomorphic with the elliptic curve

$$
y^{2}+y=x^{3}+x^{2}-44704 x-3655907 .
$$

The functions $F_{n}$ on this curve may be given by

$$
\begin{aligned}
F_{4}= & x^{2}-1208 x-227671+91 y \\
F_{9}= & \left(8 x^{3}-384 x^{2}-13656232 x-678909344\right) y \\
& -\left(1015 x^{4}+770584 x^{3}+163098512 x^{2}+29785004488 x+2319185361392\right), \\
F_{14}= & 8 x^{7}+400071 x^{6}-343453068 x^{5}-238003853192 x^{4} \\
& -116011622641292 x^{3}-15704111899877744 x^{2} \\
& -95316727595264672 x+53553894620234333456 \\
& -\left(8428 x^{5}+19974360 x^{4}+18880768004 x^{3}+4128079708928 x^{2}\right. \\
& +335969653582304 x+17681731246686360) y
\end{aligned}
$$

satisfying

$$
64 F_{4}^{7}-343 F_{9}^{3}=F_{14}^{2}
$$

and then

$$
t=\frac{F_{14}^{2}}{64 F_{4}^{7}}=1-\frac{343 F_{9}^{3}}{64 F_{4}^{7}}
$$

Proof: With a symbolic algebra package one may readily confirm the identity among the $F_{n}$, and might even feasibly (albeit not happily) verify the Galois group by following the 28 preimages of a point on the $t$-line as that point loops around $t=0$ and $t=1$; this would suffice to prove the theorem (since the cover $\mathcal{X}_{0}(3) \rightarrow \mathcal{X}(1)$ is determined by its Galois group and ramification behavior), but would not explain the provenance of the formulas. We thus devote most of the proof to the computation of the $F_{n}$.

We begin by observing that our proofs of Propositions 1 and 2 used the ramification behavior of the cover $\mathcal{X}_{0}(3) \rightarrow \mathcal{X}(1)$, but not its Galois group or the Shimura-curve structure. This will remain true in the rest of our computation 2 We thus show that the ramification behavior uniquely determines the degree-28 cover. In particular this yields the following purely group-theoretical statement (that we could also have checked directly): any permutations $\sigma_{2}, \sigma_{3}, \sigma_{7}$ of 28 letters with cycle structure $2^{14}, 3^{9} 1,7^{4}$ such that $\sigma_{2} \sigma_{3} \sigma_{7}=1$ are equivalent under conjugation in $S_{28}$ with our generators of $\mathrm{PSL}_{2}\left(\mathbf{F}_{27}\right)$, and that we do not have to verify the Galois group as suggested in the previous paragraph.

Our strategy is to first find the cover modulo the prime 29, which occurs in one of the formulas in Proposition 2(ii), and then use Newton's method over $\mathbf{Q}_{29}$

\footnotetext{
2 We shall retain the notation $\mathcal{X}_{0}(3)$ for the curve and $w_{3}$ for the involution, rather than introduce separate notations for an elliptic curve that we have not yet identified with $\mathcal{X}_{0}(3)$ and the multiplication-by- $(-1)$ map on the curve.
} 
to compute a lift of the coefficients to sufficient 29 -adic precision to recognize them as rational numbers. The prime 29 is large enough to guarantee good reduction of any Belyi cover of degree 28: if a Belyi cover has bad reduction at some prime $p$ then the normal closure of the cover has a Galois group whose order is a multiple of $p$ Bec.

We may assume that each $F_{n}(n=14,9,4)$ is scaled so that it has 29-adically integral coefficients and does not reduce to zero mod 29. In characteristic 29, the second function in Proposition 2(ii) simplifies to $6 F_{4} F_{9}^{\prime \prime} / F_{9}$. Again we use the fact that $F_{4}, F_{9}$ have no common zeros to conclude that $\xi:=F_{9}^{\prime \prime} / F_{9}$ is regular away from $P_{0}$. At $P_{0}$, we know that $F_{9}$ has a pole of order 9 ; thus $F_{9}^{\prime \prime}$ has a pole of order 11, and $\xi$ has a double pole. Since $\xi$ is regular elsewhere, it follows that $\xi$ is invariant under $w_{3}$.

We claim that $F_{9}$ is anti-invariant, that is, that $w_{3}\left(F_{9}\right)=-F_{9}$. Indeed the differential equation $f^{\prime \prime}=\xi f$ satisfied by $F_{9}$ must also hold for the invariant and anti-invariant parts of $F_{9}$, call them $F_{9}^{+}, F_{9}^{-}$. Now $F_{9}^{-}$has a pole of order 9 at $P_{0}$. If $F_{9}^{+}$is not identically zero then its valuation at $P_{0}$ is $-d$ for some $d \in\{0,2,4,6,8\}$; comparing leading coefficients in the local expansion about $P_{0}$ of $F_{9}^{+\prime \prime}=\xi F_{9}^{+}$and $F_{9}^{-\prime \prime}=\xi F_{9}^{-}$, we obtain $9 \cdot 10 \equiv d(d+1) \bmod 29$, which is impossible. Hence $F_{9}^{+}=0$ as claimed. (We could also have obtained $F_{9}^{+}=0$ by arguing that $t=1$ is the only supersingular point on $\mathcal{X}(1) \bmod 29$, whence the zeros of $F_{9}$, which are the preimages of $t=1$, must be permuted by $w_{3}$. But this would break our promise not to rely on the modular provenance of the cover.)

Now let $y^{2}=x^{3}+a x+b$ be a (narrow) Weierstrass equation for $\mathcal{X}_{0}(3)$, and choose $\omega=d x / y$. Then our derivation $f \mapsto f^{\prime}$ is characterized by $x^{\prime}=y$ and $y^{\prime}=\left(3 x^{2}+a\right) / 2$. For the rest of this paragraph we calculate modulo 29 . We have seen that $F_{9}$ is a scalar multiple of $\left(x^{3}+s_{1} x^{2}+s_{2} x+s_{3}\right) y$ for some $s_{1}, s_{2}, s_{3} \in \overline{\mathbf{F}_{29}}$. Equating coefficients in $F_{9}^{\prime \prime}=\xi F_{9}$, we find first that $\xi=8 x+6 s_{1}$, then that $s_{2}=-12 s_{1}^{2}-8 a$ and $s_{3}=7 b-3 s_{1}^{3}-a s_{1}$, and finally that $s_{1} b+s_{1}^{4}+9 a s_{1}^{2}+9 a^{2}=$ 0 . We have also seen that $F_{4}$ is a scalar multiple of $x^{2}+t_{1} y+t_{2} x+t_{4}$ for some $t_{1}, t_{2}, t_{4} \in \overline{\mathbf{F}_{29}}$. Using Proposition 2(i) we compute $F_{14}$ and $F_{4}^{6}$ up to scaling in terms of $s_{1}, a, b$ and the $t_{i}$, and compare with $\left(x^{2}+t_{1} y+t_{2} x+t_{4}\right)^{6}$. After matching the leading (degree-24) coefficients ${ }^{3}$ we find that the degree-23 coefficients agree identically, but in degree 22 we find $t_{2}=11\left(t_{1}^{2}-s_{1}\right)$, and the degree 21 comparison yields $t_{1}=0$ or $t_{1}^{2}=5 s_{1}$. We cannot have $t_{1}=0$, for then $F_{4}$ would be even, and then so would $F_{14}$ (since $F_{14}$ is proportional to $3 F_{4} F_{9}^{\prime}-7 F_{4}^{\prime} F_{9}$ ), which is impossible since the nonzero odd function $F_{9}^{3}$ is a linear combination of $F_{4}^{7}$ and $F_{14}^{2}$. Therefore $t_{1}^{2}=5 s_{1}$. Comparing the next few coefficients in our two expressions for $F_{4}^{6}$, we learn that $t_{4}=s_{1}^{2}+3 a$ and $a=9 s_{1}^{2}$. This completes the determination of our cover mod 29 up to scaling. For instance, we may use the equation $y^{2}=x^{3}+9 x+1$ for $\mathcal{X}_{0}(3)$ over $\overline{\mathbf{F}_{29}}$, and then check that

$$
F_{4}=x^{2}+11 y-14 x-1, \quad F_{9}=\left(x^{3}+x^{2}+3 x-5\right) y,
$$

\footnotetext{
3 The "degree" of a polynomial in $x, y$ is the order of its pole at $P_{0}$. The vector space of rational functions on $\mathcal{X}_{0}(3)$ that are regular away from $P_{0}$ has basis $\left\{x^{i} \mid i=\right.$ $0,1,2, \ldots\} \cup\left\{x^{i} y \mid i=0,1,2, \ldots\right\}$; the monomials $x^{i}, x^{i} y$ have degrees $2 i, 2 i+3$.
} 
$F_{14}=\left(x^{7}-14 x^{6}-5 x^{5}-9 x^{4}-10 x^{3}+2 x^{2}+10 x-7\right)-\left(8 x^{5}-x^{3}-3 x^{2}+x+3\right) y$ satisfy the identity $F_{4}^{7}-6 F_{9}^{3}=F_{14}^{2}$.

We now use Hensel's lemma to show that there is a unique such identity over $\mathbf{Z}_{29}$. To do this we regard $\alpha F_{4}^{7}+\beta F_{9}^{3}=F_{14}^{2}$ as an overdetermined system of equations in $a, b, \alpha, \beta$, and the coefficients of $F_{4}$ and $F_{9}$. More precisely, we eliminate the ambiguity in the various choices of scalar multiples by requiring that $F_{4}$ and $F_{9}$ each have leading coefficient 1 , setting $b=9 a$, and defining $F_{14}$ by the formula $3 F_{4} F_{9}^{\prime}-7 F_{4}^{\prime} F_{9}$ from Proposition $2(\mathrm{i})$. (Any elements of $\mathbf{Z}_{29}$ that reduce to $1 \bmod 29$ would do for the leading coefficients, as would any element of $\mathbf{Z}_{29}$ that reduces to $9 \bmod 29$ for $b / a$.) We include in our equations the $x^{i}$ coefficients of $F_{9}(i=0,1,2,3,4)$, which were known to vanish mod 29 but not in $\mathbf{Z}_{29}$. By our analysis thus far, this system of equations has a unique solution mod 29. We compute the Jacobian matrix of our system of equations at that solution, and verify that this matrix has full rank. Therefore the solution lifts uniquely to $\mathbf{Z}_{29}$ by Hensel's lemma. This is also the unique solution over $\mathbf{Q}_{29}$, because 29 is a prime of good reduction as noted above.

It remains to recover the coefficients as rational numbers. We compute them mod $29^{128}$ by applying a Newton iteration 7 times 29 -adically. (At each step, instead of computing the derivative $\bmod 29^{2^{n-1}}$ symbolically, we approximate each partial derivative by evaluating the function mod $29^{2^{n}}$ at two points that differ by $29^{2^{n-1}}$ times the corresponding unit vector. We also streamline the computation by requiring at each step that only the $x^{i}$ coefficients match, ignoring the $x^{i} y$ terms; this suffices because the resulting submatrix of the Jacobian matrix still has full rank mod 29.) We then use 2-dimensional lattice reduction to guess the rational numbers represented by those 29-adic approximations, and verify the guess by checking that the resulting $F_{4}^{7}, F_{9}^{3}, F_{14}^{2}$ are $\mathbf{Q}$-linearly dependent. This completes the determination of the cover $\mathcal{X}_{0}(3) \rightarrow \mathcal{X}(1)$ and the proof that it is characterized by its degree and ramification behavior. Finally we bring the curve $y^{2}=x^{3}+a x+b$ to minimal form and replace $F_{4}, F_{9}, F_{14}$ by the smallest rational multiples that eliminate the denominators of those rational functions. This yields the formulas in Theorem 1. $\diamond$

In the same way that we proved $F_{9}$ is an odd function mod 29 , we can use the other two formulas in Proposition 2(ii) to prove that $F_{4}$ and $F_{14}$ are even mod 13 and 43 respectively. This is confirmed by Theorem 1: the terms containing $y$ in $F_{4}$ and $F_{14}$ are divisible by 13 and 43 respectively. The corresponding result for $F_{9} \bmod 29$ is obscured by the minimal form of $\mathcal{X}_{0}(3)$, which makes it harder to recognize odd functions; but $F_{9}$ can be seen to be congruent mod $2 y+1$ to a multiple of 29 , as expected.

\subsection{Application: the CM point of discriminant -11}

In [E2, 5.3], we noted that $\mathcal{X}(1)$ has a unique CM point of discriminant -11 , and therefore that this point has rational $t$-coordinate. We then described highprecision numerical computations that strongly suggest this $t$-coordinate is

$$
\frac{88983265401189332631297917}{45974167834557869095293}=\frac{7^{3} 43^{2} 127^{2} 139^{2} 307^{2} 659^{2} 11}{3^{3} 13^{7} 83^{7}},
$$


with $t-1$ having numerator $2^{9} 29^{3} 41^{3} 167^{3} 281^{3}$. But we could not prove that this is correct. Our formulas for the cover $\mathcal{X}_{0}(3) \rightarrow \mathcal{X}(1)$ now let us do this:

Corollary to Theorem 1. The CM-11 point on $\mathcal{X}(1)$ has t-coordinate equal $7^{3} 43^{2} 127^{2} 139^{2} 307^{2} 659^{2} 11 / 3^{3} 13^{7} 83^{7}$, and lies under the two points of $\mathcal{X}_{0}(3)$ with $(x, y)=(-10099 / 64,-1 / 2 \pm 109809 \sqrt{-11} / 512)$.

Proof: The CM-11 point has two preimages in $\mathcal{X}_{0}(3)$ that are switched by $w_{3}$, corresponding to a pair of "3-isogenies" between (the abelian variety parametrized by) that point and itself, namely the pair $(-1 \pm \sqrt{-11}) / 2$ of norm-3 elements of the endomorphism ring. A point of $\mathcal{X}(1)$ is 3 -isogenous to itself if and only if its $t$-coordinate satisfies $t=t(P)=t\left(w_{3} P\right)$ for some point $P$ on $\mathcal{X}_{0}(3)$. Equivalently, $P$ is either a common pole of the functions $t(P), t\left(w_{3} P\right)$ on $\mathcal{X}_{0}(3)$ or a zero of the rational function $t(P)-t\left(w_{3} P\right)$ on that curve. The former cannot happen here because by our formulas $F_{4}$ and $F_{4} \circ w_{3}$ have no common zeros. Since $t(P)-t\left(w_{3} P\right)$ is an odd function on $\mathcal{X}_{0}(3)$, we easily locate its zeros by writing it as $2 y+1$ times a rational function of $x$. We find that the latter function vanishes at $x=\infty, x=-1097 / 8, x=-10099 / 64$, and the roots of four irreducible polynomials of degree 3 and two irreducibles of degree 6 . At $x=\infty$ we have $P=P_{0}$ and $t=1$. At $x=-1097 / 8$ we have $y=-1 / 2 \pm 6615 \sqrt{-2} / 32$, and calculate that $t=1092830632334 / 1694209959$, which we already identified as the CM-8 point by a similar computation with the curve $\mathcal{X}_{0}(2)$ E2, p.39]. The irrational values of $x$ that are roots of the irreducible polynomials of degree 3 and 6 yield irrational $t$-values of the same degree. Hence the CM-11 point, being rational, must have $x=-10099 / 64$, for which we calculate the values of $y$ and $t$ given in the statement of the Corollary. $\diamond$

The CM-8 point also has an element of norm 3 in its endomorphism ring, namely $1+\sqrt{-2}$. The fact that the CM points with $x=-10099 / 64$ have discriminant -11 also follows from the fact that their $y$-coordinates are conjugates over $\mathbf{Q}(\sqrt{-11})$. The irrational values of $x$ have degrees 1 and 2 over $K$; in particular, the cubic irrationalities yield four $\operatorname{Gal}(K / \mathbf{Q})$-orbits of $\mathrm{CM}$ points. Taking $c=2 \cos (2 \pi / 7)$, we find that these CM points have $x$-coordinates $189 c-19$, $-\left(189 c^{2}+567 c+397\right),-2\left(756 c^{2}+1701 c+671\right),-2\left(3591 c^{2}+8127 c+2939\right)$, and their $\operatorname{Gal}(K / \mathbf{Q})$ conjugates. We determine their CM fields as the fields of definition of the points' $y$-coordinates: they are the fields obtained by adjoining to $K$ the square roots of the totally negative algebraic integers $c^{2}+2 c-7$, $c-6,-\left(3 c^{2}+2 c+3\right)$, and $c^{2}-2 c-11$, of norms $-167,-239,-251$, and -491 respectively.

\section{The curve $\mathcal{X}_{1}(3)$, and its Jacobian $\mathcal{J}_{1}(3)$}

The tables $\mathrm{BK} \mathrm{Cr}$ of elliptic curves of low conductor indicate that the curve 147-B2(J), which we identified with $\mathcal{X}_{0}(3)$, is 13 -isogenous with another elliptic curve over Q, namely 147-B1(I): [0,1,1,-114,473]. Now 13-isogenies between elliptic curves over $\mathbf{Q}$, though not hard to find (via a rational parametrization of the classical modular curve $\left.\mathrm{X}_{0}(13)\right)$, are rare: this 13 -isogeny, and its twist by $\mathbf{Q}(\sqrt{-7})$ curves $147-\mathrm{C} 1(\mathrm{~A})$ and $147-\mathrm{C} 2(\mathrm{~B})]$, are the only examples up to 
conductor $200 \mathrm{BK}, 4$ even up to conductor 1000, the only other examples are the twists of these 13-isogenies by $\mathbf{Q}(\sqrt{-3})$, which appear at conductor 441 . The fact that $\mathcal{X}_{0}(3)$ admits a rational 13-isogeny thus seemed a remarkable coincidence. Mark Watkins Wa observes that this curve 147-B1(I) has an even more unusual property: not only is there a 13-isogeny from this curve to $\mathcal{X}_{0}(3)$, but the kernel of the isogeny consists of points rational over $K$. Whereas the classical modular curve $\mathrm{X}_{0}(13)$, which parametrizes (generalized) elliptic curves with a rational 13 -isogeny, has genus 0 , the curve $\mathrm{X}_{1}(13)$, which parametrizes (generalized) elliptic curves with a rational 13-torsion point, has genus 2. Thus by Mordell-Faltings $\mathrm{X}_{1}(13)$ has only finitely many $K$-rational points. Hence there are only finitely many isomorphism classes of elliptic curves defined over $K$, let alone over $\mathbf{Q}$, with a $K$-rational 13-torsion point - and we have found one of them 5 by computing the Jacobian of the Shimura modular curve $\mathcal{X}_{1}(3)$ !

These observations are explained by considering $\mathcal{X}_{1}(3)$. This curve, like $\mathcal{X}_{0}(3)$, is defined over $\mathbf{Q}$, and the cyclic cover $\mathcal{X}_{1}(3) \rightarrow \mathcal{X}_{0}(3)$ has degree $\left(3^{3}-1\right) / 2=13$. This cover must be unramified, because the only elliptic point on $\mathcal{X}_{0}(3)$ is of order 3 , which is coprime to 13 . Hence $\mathcal{X}_{1}(3)$ has genus 1 . It does not quite follow that $\mathcal{X}_{1}(3)$ is isomorphic over $\mathbf{Q}$ with the 13 -isogenous elliptic curve $147-\mathrm{B} 1(\mathrm{I})$, because $\mathcal{X}_{1}(3)$ need not have any $\mathbf{Q}$-rational point. However, the Jacobian $\mathcal{J}_{1}(3)$ of $\mathcal{X}_{1}(3)$ is an elliptic curve, and is also 13 -isogenous with $\mathcal{X}_{0}(3)$ because the cover $\mathcal{X}_{1}(3) \rightarrow \mathcal{X}_{0}(3)$ induces a map of the same degree from $\mathcal{J}_{1}(3)$ to $\mathcal{X}_{0}(3)$. Since the elliptic curve $147-\mathrm{B} 1(\mathrm{I})$ is the only one 13 -isogenous with $\mathcal{X}_{0}(3)$ over $\mathbf{Q}$, we conclude that it is isomorphic with $\mathcal{J}_{1}(3)$. Furthermore, the geometric Galois group of the cover $\mathcal{X}_{1}(3) / \mathcal{X}_{0}(3)$ is canonically $k^{*} /\{ \pm 1\}$, where $k$ is the residue field of the prime above 3 in $K$. Working over $K$, we can choose a generator for this group, which acts on $\mathcal{X}_{1}(3)$ by translation by some element of $\mathcal{J}_{1}(3)$, and this element is a 13-torsion point that generates the kernel of the isogeny from $\mathcal{J}_{1}(3)$ to $\mathcal{X}_{0}(3)$.

This is all quite reminiscent of the situation for the classical modular curves $\mathrm{X}_{0}(11), \mathrm{X}_{1}(11)$, which are 5-isogenous elliptic curves, with the kernel of the isogeny $\mathrm{X}_{1}(11) \rightarrow \mathrm{X}_{0}(11)$ canonically isomorphic with $\mathbf{F}_{11}^{*} /\{ \pm 1\}$ and thus con-

4 Table 4 in BK], which gives all curves of conductor $2^{a} 3^{b}$, gives several other examples of conductor $20736=2^{8} 3^{4}$, all related by quadratic twists.

5 Not, however, the unique one. The relevant twist of $\mathrm{X}_{1}(13)$ by $K$ is isomorphic with the curve $y^{2}=4 x^{6}+8 x^{5}+37 x^{4}+74 x^{3}+57 x^{2}+16 x+4$, which inherits from $\mathrm{X}_{1}(13)$ a 3 -cycle generated by $(x, y) \mapsto\left(-x^{-1}-1, y / x^{3}\right)$. Rational points related by this 3-cycle and/or the hyperelliptic involution parametrize the same curve, with a different choice of generator of the torsion subgroup. The orbit of $\mathbf{Q}$-rational points with $x \in 0,-1, \infty$ yields our curve $\mathcal{J}_{1}(3)$. A computer search finds that there is at least one other orbit, represented by $(x, y)=(17 / 16,31585 / 2048)$, which leads to a second elliptic curve over $\mathbf{Q}$ with a $K$-rational 13-torsion point. We calculate that this is the curve with coefficients

$[0,1,1,-69819305996260175838254,7100867435908988429025874812520367]$

and conductor $8480886141=3 \cdot 7^{2} \cdot 13 \cdot 251 \cdot 17681$. 
sisting of Q-rational 5-torsion points. Also, $\mathrm{X}_{1}(11)$ has considerably smaller height (visible in its smaller coefficients) and discriminant than $\mathrm{X}_{0}(11)$, as does $\mathcal{J}_{1}(3)$ compared with $\mathcal{X}_{0}(3)$. The comparison becomes even clearer if we work with models of these curves minimal over $K$, where the additive reduction at the rational prime 7 becomes good reduction at the prime $(2-c)$ of $K$ above 7 .

Now for the classical modular curves the fact that $\mathrm{X}_{1}(11)$ has a simpler equation than $\mathrm{X}_{0}(11)$ illustrates a general phenomenon noted in Ste]: the minimal height in an isogeny class of elliptic curves over $\mathbf{Q}$ is conjecturally attained by the optimal quotient of the Jacobian of $\mathrm{X}_{1}(N)$, not $\mathrm{X}_{0}(N)$ (unless the $\mathrm{X}_{1}$ - and $\mathrm{X}_{0}$-optimal quotients coincide). Does this always happen also for Shimura curves? We observed the same behavior in several other cases, one of which appears in the next section. But there are no extensive tables of optimal quotients of Jacobians of Shimura curves on which one might test such a conjecture. Although Vatsal [Va, Thm. 1.11] has proven Stevens' conjecture for curves with a rational $\ell$-isogeny for prime $\ell \geq 7$, his methods cannot apply in our setting (even though all our curves have a suitable isogeny) because they rely on congruences between $q$-expansions of modular forms, a tool that is not available to us in the Shimura-curve setting.

\section{Some other Shimura curves of genus 1}

Watkins notes several other examples of curves in $\mathrm{Cr}$ that behave similarly for other choices of cyclic cubic fields $K$, and asks whether they, too, can be explained as Shimura modular curves or Jacobians. We checked that this is the case for at least one of these examples, at conductor $162=2 \cdot 9^{2}$. In this section, we outline this computation and describe a 7 -isogeny in conductor $338=2 \cdot 13^{2}$ that should also involve Shimura modular curves.

For the curves of conductor 162, we start with a quaternion algebra over $K_{9}=\mathbf{Q}(\cos 2 \pi / 9)$ ramified at two of the three real places of $K_{9}$ and at none of its finite primes. The resulting modular group $\Gamma(1)$ is again a triangle group, this time with signature $(2,3,9)$ rather than $(2,3,7)$ (see [T], class XI). Since the rational prime 2 is inert in $K_{9}$, we have modular curves $\mathcal{X}_{0}(2)$ and $\mathcal{X}_{1}(2)$ with geometric Galois group $\mathrm{PSL}_{2}\left(\mathbf{F}_{8}\right)$ over the rational curve $\mathcal{X}(1)$. We calculate that here $\mathcal{X}_{0}(2)$ already has genus 1 . Since the Belyi map $\mathcal{X}_{0}(2) \rightarrow \mathcal{X}(1)$ has degree as low as $8+1=9$, we can find its coefficients with little difficulty even without resorting to the methods we used to obtain the equations in Theorem 1. We place the elliptic points of $\mathcal{X}(1)$ at $t=1,0, \infty$. Then $t$ is a rational function of degree 9 on $\mathcal{X}_{0}(2)$ with a ninth-order pole, which we use as the base point $P_{0}$ to give $\mathcal{X}_{0}(2)$ the structure of an elliptic curve. The zero divisor of $t$ is then $3 D_{3}$ for some divisor $D_{3}$ of degree 3 . Hence $D_{3}-3\left(P_{0}\right)$ is a 3 -torsion divisor on $\mathcal{X}_{0}(2)$. By computing the divisor of $d t$ as in the proof of Proposition 1(i), we see that the corresponding 3 -torsion point on $\mathcal{X}_{0}(2)$ is also the simple zero of $t-1$. In particular, this 3 -torsion point cannot be trivial. (This fact could also be deduced by noting that if the 3 -torsion point were trivial then $t$ would be the cube of a rational function on $\mathcal{X}_{0}(2)$, and so could not have Galois group 
$\mathrm{PSL}_{2}\left(\mathbf{F}_{8}\right)$.) The general elliptic curve with a rational 3-torsion point has the form $y^{2}+a_{1} x y+a_{3} y=x^{3}$, with the torsion point at $(x, y)=(0,0)$. Solving for $\left(a_{3}: a_{1}^{3}\right)$ and the coefficients of $t$, we soon find a model of $\mathcal{X}_{0}(2)$ with $\left(a_{1}, a_{3}\right)=(15,128)$ and $t=\left(y-x^{2}-17 x\right)^{3} /\left(2^{14} y\right)$, and with the double roots of $t-1$ occurring at the zeros of $(x+9) y+6 x^{2}+71 x$ other than $(0,0)$. Reducing $\mathcal{X}_{0}(2)$ to standard minimal form, we find the curve 162-B3(I): [1, -1, 1, -95, -697]. As expected, this curve attains its 3 -adic potential good reduction over $K_{9}$. The involution $w_{2}$ is the unique involution of this elliptic curve that fixes the simple zero $(0,-128)$ of $t-1$. For instance, $w_{2}$ sends the point at infinity to the other 3 -torsion point $(0,0)$, at which $t=-17^{3} / 2^{7}$. Hence $t=-17^{3} / 2^{7}$ is a $\mathrm{CM}$ point on $\mathcal{X}(1)$, the unique point 2-isogenous with the elliptic point $t=\infty$. Solving $t(P)=t\left(w_{2}(P)\right)$ yields the further CM point $t=17^{3} 5^{3} / 2^{6}$, which must have CM field $K_{9}(\sqrt{-7})$ because $P$ and $w_{2}(P)$ are conjugate over $\mathbf{Q}(\sqrt{-7})$.

Besides the known 3-torsion point, we find that $\mathcal{X}_{0}(2)$ also has a 7 -isogeny with the curve $162-\mathrm{B} 1(\mathrm{G})$ : $[1,-1,1,-5,5]$. It is known (see for instance the "remarks on isogenies" in [BK] that the isogeny class of this curve is the unique one, up to quadratic twist, with both a 3- and a 7-isogeny over $\mathbf{Q}$. We have already accounted for the 3 -torsion point using the ramification behavior of the map $\mathcal{X}_{0}(2) \rightarrow \mathcal{X}(1)$. The 7 -isogeny is again explained using $\mathcal{X}_{1}$. This time the cyclic cover $\mathcal{X}_{1}(2) \rightarrow \mathcal{X}_{0}(2)$ has degree $2^{3}-1=7$, and the only elliptic point of $\mathcal{X}_{0}(2)$ is the simple zero of $t-1$, which has order 2 . Since $\operatorname{gcd}(2,7)=1$, the cyclic cover is again unramified, and $\mathcal{J}_{1}(2)$ must be an elliptic curve 7 -isogenous with $\mathcal{X}_{0}(2)$. Hence $\mathcal{J}_{1}(2)$ is isomorphic with the elliptic curve $162-\mathrm{B} 1(\mathrm{G})$ : $[1,-1,1,-5,5]$. Also as before, the kernel of the isogeny must consist of points rational over $K_{9}$, and again Watkins confirms [Wa that this is the case for this curve. This time it turns out $\mathcal{J}_{1}(2)$ is the unique elliptic curve over $\mathbf{Q}$ with the correct torsion structure: a 3-torsion point over $\mathbf{Q}$, and a 7-torsion point over $K_{9}$ that is proportional to its Galois conjugates. Again we note that it is $\mathcal{J}_{1}(2)$, not $\mathcal{X}_{0}(2)$, that has the smaller discriminant and height.

The next case is the cyclic cubic field $K_{13}$ of discriminant $169=13^{2}$. Once more we use a quaternion algebra over this field that is ramified at two of its three real places and at none of the finite primes. By Shimizu's formula (Sh2, Appendix], quoted in [T, p.207]), the resulting curve $\mathcal{X}(1)$ has hyperbolic area

$$
\frac{1}{16 \pi^{6}} d_{K_{13}}^{3 / 2} \zeta_{K_{13}}(2)=-\frac{1}{2} \zeta_{K_{13}}(-1)
$$

Since $K_{13}$ is cyclotomic, we can compute $\zeta_{K_{13}}(2)$ or $\zeta_{K_{13}}(-1)$ by factoring $\zeta_{K}$ as a product of the Riemann zeta function and two Dirichlet $L$-series. We find that $\mathcal{X}(1)$ has normalized hyperbolic area $1 / 6$. Since $\Gamma(1)$ is not on Takeuchi's list of triangle groups [T, the curve $\mathcal{X}(1)$ must have at least four elliptic points, or positive genus and at least one elliptic point. The only such configuration that attains an area as small as $1 / 6$ is genus zero, three elliptic points of order 2 , and one of order 3. Again we use the prime of $K$ above the inert rational prime 2 to construct modular curves $\mathcal{X}_{0}(2)$ and $\mathcal{X}_{1}(2)$ with geometric Galois group $\mathrm{PSL}_{2}\left(\mathbf{F}_{8}\right)$ over the rational curve $\mathcal{X}(1)$. In the degree-9 cover $\mathcal{X}_{0}(2) \rightarrow \mathcal{X}(1)$, the elliptic point of order 3 has 3 triple preimages, and each of the order- 2 points has 
one simple and four double preimages. Hence $\mathcal{X}_{0}(2)$ has genus 1 by RiemannHurwitz. Again the cyclic cover $\mathcal{X}_{1}(2) \rightarrow \mathcal{X}_{0}(2)$ has degree 7 , relatively prime to the orders of the elliptic points on $\mathcal{X}_{0}(2)$, so $\mathcal{X}_{1}(2)$ also has genus 1.

In this setting it is not clear that either $\mathcal{X}_{0}(2)$ or $\mathcal{X}_{1}(2)$ is an elliptic curve over $\mathbf{Q}$, since neither curve is forced to have a $\mathbf{Q}$-rational divisor of degree 1. (The elliptic points of order 2 may be Galois conjugates, not individually rational over Q.) But we can still consider the Jacobians $\mathcal{J}_{0}(2)$ and $\mathcal{J}_{1}(2)$, which are elliptic curves 7-isogenous over $\mathbf{Q}$, with the kernel of the 7-isogeny $\mathcal{J}_{1}(2) \rightarrow \mathcal{J}_{0}(2)$ consisting of points rational over $K_{13}$. Watkins suggests, by analogy with the cases of conductor 147 and 162, that this 7-isogeny should connect curves of conductor $2 \cdot 13^{2}=338$. According to the tables of $\mathrm{Cr}$, there are three pairs of 7 -isogenous curves of conductor 338. Watkins computes that of the 6 curves involved in these isogenies, only 338 -B1: $[1,-1,1,137,2643]$ has 7 -torsion points rational over $K_{13}$, and thus proposes this curve as $\mathcal{J}_{1}(2)$, and the 7 -isogenous curve 338-B2: $[1,-1,1,-65773,-6478507]$ as $\mathcal{J}_{0}(2)$. This must be correct, since these Jacobians should again have multiplicative reduction at 2 and good reduction at all primes of $K_{13}$ other than 2 , whence their conductor must be $2 \cdot 13^{2}$ (there being no curve of conductor 2 over $\mathbf{Q}$ ). But here I have not obtained an explicit rational function or even determined the cross-ratio of the elliptic points on $\mathcal{X}(1)$, which would be quite a demanding computation using the methods of [E2].

Acknowledgements. This work was supported in part by NSF grants DMS0200687 and DMS-0501029. Symbolic and numerical computations were assisted by the computer packages PARI/GP $\mathrm{P}$ and MAXIMA. I am also grateful to Mark Watkins for the reference to $\mathrm{Va}$ and other relevant correspondence $\mathrm{Wa}$, to Watkins and the referees for careful readings of and corrections to earlier drafts of this paper, to the referees for suggesting several other bibliographical sources, and to Benedict Gross for the remarks in footnote 1

\section{References}

ACGH. Arbarello, E., Cornalba, M., Griffiths, P.A., Harris, J.: Geometry of Algebraic Curves, Vol. I. New York: Springer, 1985.

Bec. Beckmann, S.: Ramified primes in the field of moduli of branched coverings of curves, J. of Algebra 125 (1989), 236-255.

Bel. Belyi, G.V.: On the Galois extensions of the maximal cyclotomic field, Izv. Akad. Nauk SSSR 43 (1979), 267-76 (= Math. USSR Izv. 14 (1980), 247256); see also: A new proof of the three-point theorem, Mat. Sb. 193 (2002) \#3, 21-24 (= Sb. Math. 193 (2002) \#3-4, 329-332).

BK. Birch, B.J., Kuyk, W., ed.: Modular Functions of One Variable IV. Lect. Notes in Math. 476, 1975.

Ca. Carayol, H.: Sur la mauvaise réduction des courbes de Shimura, Compositio Math. 59 (1986) \#2, 151-230.

Cr. Cremona, J.E.: Algorithms for modular elliptic curves. Cambridge University Press, 1992. 
DN. Doi, K., Naganuma, H.: On the algebraic curves uniformized by arithmetical automorphic functions, Annals of Math. (2) 86 (1967), 449-460.

E1. Elkies, N.D.: Explicit modular towers, pages 23-32 in Proceedings of the Thirty-Fifth [1997] Annual Allerton Conference on Communication, Control and Computing (T. Başar and A. Vardy, eds.; Univ. of Illinois at UrbanaChampaign, 1998) = http://arXiv.org/abs/math.NT/0103107.

E2. Elkies, N.D.: Shimura curve computations, pages 1-47 in Algorithmic number theory (Portland, OR, 1998; J. Buhler, ed.; Lect. Notes in Computer Sci. \#1423; Berlin: Springer, 1998) = http://arXiv.org/abs/math.NT/0005160.

E3. Elkies, N.D.: The Klein quartic in number theory, pages 51-102 in The Eightfold Way: The Beauty of Klein's Quartic Curve (S.Levy, ed.; Cambridge Univ. Press, 1999); online at http://www.msri.org/publications/books/Book35/.

F1. Fricke, R.: Über den arithmetischen Charakter der zu den Verzweigungen $(2,3,7)$ und $(2,4,7)$ gehörenden Dreiecksfunctionen, Math. Ann. 41 (1893), 443-468.

F2. Fricke, R.: Entwicklungen zur Transformation fünfter und siebenter Ordnung einiger specieller automorpher Functionen, Acta Math. 17 (1893), 345-395.

F3. Fricke, R.: Ueber eine einfache Gruppe von 504 Operationen, Math. Ann. 52 (1899), 321-339.

GR. González, J., Rotger, V.: Equations of Shimura curves of genus 2 (http://arXiv.org/abs/math.NT/0312434), to appear in International Math. Research Letters.

H. Hurwitz, A.: Über algebraische Gebilde mit eindeutigen Transformationen in sich, Math. Annalen 41 (1893), 403-442.

K. Kurihara, A.: On some examples of equations defining Shimura curves and the Mumford uniformization, J. Fac. Sci. Univ. Tkyo, Sec. IA 25 (1979), 277-301.

Ma. $\quad$ Macbeath, A.M.: On a curve of genus 7, Proc. LMS 15 (1965), 527-542.

Mu. Mumford, D.: Abelian Varieties. London: Oxford Univ. Press, 1970.

P. PARI/GP, versions 2.1.1-4, Bordeaux, 2000-4, http://pari.math.u-bordeaux.fr .

Se. $\quad$ Serre, J.-P.: Topics in Galois Theory. Boston: Jones and Bartlett 1992.

Sh1. Shimura, G.: Construction of class fields and zeta functions of algebraic curves, Ann. of Math. 85 (1967), 58-159.

Sh2. Shimizu, H.: On zeta functions of quaternion algebras, Ann. of Math. 81 (1965), 166-193.

Ste. Stevens, G.: Stickelberger elements and modular parametrizations of elliptic curves, Invent. Math. 98 (1989) \#1, 75-106.

Str. Streit, M.: Field of definition and Galois orbits for the Macbeath-Hurwitz curves, Arch. Math. 74 (2000) \#5, 342-349

T. Takeuchi, K.: Commensurability classes of arithmetic triangle groups, J. Fac. Sci. Univ. Tokyo 24 (1977), 201-212.

Va. Vatsal, V.: Multiplicative subgroups of $J_{0}(N)$ and applications to elliptic curves, J. Inst. Math. Jussieu 4 (2005) \#2, 281-316.

Vi. Vignéras, M.-F.: Arithmétique des Algèbres de Quaternions, Berlin: Springer, 1980 (SLN 800).

Wa. Watkins, M.: e-mail communication, 2003-2005.

Wo. Wolfart, J.: Belyi Surfaces with Many Automorphisms, pages 97-112 in Geometric Galois Actions, 1. Around Grothendieck's Esquisse d'un Programme (L. Schneps, P. Lochak, eds.; London Math. Soc. Lect. Note Series 242), Cambridge University Press, 1997. 